\title{
Surgical management of pleuro-peritoneal fistula in chronic renal failure patient-safety and effectiveness
}

\author{
Hei-Yu Matthew Chen^, Ho-Yan Howard Chan, Hoi-Ming Herman Chan, Hung-Leong Cheung \\ Department of Cardiothoracic Surgery, Queen Elizabeth Hospital, Kowloon, Hong Kong, China \\ Contributions: (I) Conception and design: HYM Chen, HYH Chan; (II) Administrative support: HYH Chan, HMH Chan; (III) Provision of study \\ materials or patients: HYH Chan; (IV) Collection and assembly of data: HYM Chen; (V) Data analysis and interpretation: HYM Chen, HYH Chan, \\ HMH Chan; (VI) Manuscript writing: All authors; (VII) Final approval of manuscript: All authors. \\ Correspondence to: Dr. Hei-Yu Matthew Chen. Department of Cardiothoracic Surgery, Queen Elizabeth Hospital, No.30 Gascoigne Road, Kowloon, \\ Hong Kong, China. Email: mattchenmailbox@gmail.com.
}

\begin{abstract}
Background: Pleuro-peritoneal fistula (PPF) is a known complication arising in patients receiving continuous ambulatory peritoneal dialysis (CAPD) as a form of renal replacement therapy with an incidence of approximately $2 \%$ (1). Previous literature has shown that the recurrence rate of non-operative management of PPF to be $\sim 45 \%$. Video-assisted thoracoscopic surgery (VATS) has been shown to be an effective and safe means of treating patients with PPF. However, to the author's knowledge, there is currently no sizeable case series that discuss the various intra-operative findings, operative techniques employed, postoperative complications, duration of peritoneal dialysis suspension and fistula recurrence in this particular patient group.

Methods: From January 2009 to January 2019, patients who underwent VATS for the repair of PPF at The Department of Cardiothoracic Surgery, Queen Elizabeth Hospital, Hong Kong were retrieved from the Clinical Data Analysis and Reporting System (CDARS). Patient's epidemiological data, comorbidities and surgical data were analyzed

Results: There were 35 patients who underwent VATS PPF repair in our series. The mean age was 60.8 years (44 to 82 years), the (54.3\%), mean operative time was 60.8 minutes (15-224 minutes). There were 8 patients $(25.8 \%)$ who suffered from recurrence of pleural effusion after re-initiation of CAPD. Concomitant use of mechanical and talc pleurodesis was statistically significant in preventing PPF recurrence with an odds ratio of 0.1201 when compared to non-operative techniques. One patient suffered from hemothorax requiring re-operation on post-operative day one. There were no 30-day mortalities.

Conclusions: VATS appears to be a safe and effective surgical treatment to prevent recurrence of continuous peritoneal dialysis associated PPF. Concomitant mechanical and talc pleurodesis appears to have additional benefit.
\end{abstract}

Keywords: Pleuro-peritoneal fistula (PPF); surgical management; pleurodesis

Submitted Dec 15, 2020. Accepted for publication Apr 02, 2021.

doi: $10.21037 /$ jtd-20-3327

View this article at: http://dx.doi.org/10.21037/jtd-20-3327

\section{Introduction}

Pleuro-peritoneal fistula (PPF) is a known complication arising in patients receiving continuous ambulatory peritoneal dialysis (CAPD) as a form of renal replacement therapy. The incidence of CAPD related PPF is approximately $2 \%$ (1). PPF manifests as chest discomfort,

\footnotetext{
^ ORCID: 0000-0003-4867-711X.
} 
shortness of breath and pleural effusion on chest radiograph. Diagnosis of CAPD associated PPF can be made clinically with compatible pleural fluid biochemistry, CT peritoneogram or by peritoneal fistulogram (2). Videoassisted thoracoscopic surgery (VATS) has been shown to be an effective and safe means of treating patients with PPF (3).

To address this entity of CAPD associated PPF and its surgical management, we aim to retrospectively analyse patients with PPF who have undergone VATS and their respective clinical parameters, operative details and clinical outcomes. In particular, we aim to review whether specific operative techniques will be associated with decreased PPF recurrence. We present the following article in accordance with the STROBE reporting checklist.

\section{Methods}

Consecutive patients who had underwent VATS for CAPD associated PPF were identified in our surgical records database between January 2009 to January 2019. Data was extracted from the "Clinical Management System" (CMS) and "Clinical Data Analysis and Reporting System" (CDARS). Clinical information was retrieved from entries made during surgical and medical follow up, surgical operation records and other relevant documentations. All patients who underwent VATS for CAPD associated PPF were included. Data analysis was performed including patients' age, gender, body weight at time of surgery, presence of diabetes mellitus (DM), concurrent use of angiotensin converting enzyme inhibitors (ACEI), duration of CAPD prior to first episode of pleural effusion, method of diagnosis of $\mathrm{PPF}$, duration of operation, intra-operative findings, surgical technique used, surgical complications requiring re-operation during same admission, duration of CAPD suspension, time between surgical repair and reinitiation of CAPD, PPF recurrence, time to recurrence, need for surgical reintervention and 30 day mortality. Patients who did not receive CAPD after the surgery were excluded from the analysis pertaining to PPF recurrence.

Observations were made by a single data collector in order to decrease reporting bias and to ensure standardization of the method of data extraction and entry. Surgeons who performed the operations were not allowed to participate in the data collection process.

The authors acknowledge that there is a relative scarcity of patients with CAPD associated PPF at each cardiothoracic centre. As such this may complicate the discerning of useful information from providing insight into our clinical practice. Hence, the authors extrapolated data from Chow et al. (1) which described the recurrence rates of CAPD associated PPF by interruption of CAPD for 1-4 months followed by re-initiation alone as well as chest tube pleurodesis (Appendix 1). The aforementioned data was used as a baseline for comparison of PPF recurrence and statistical analysis.

\section{Statistical analysis}

Continuous variables were analysed by linear regression analysis. Categorical variables were analysed by Fisher's exact test. $\mathrm{P}$ value of $<0.05$ was taken as statistically significant. Odds ratios with corresponding confidence intervals were used in subgroup analysis and in evaluation of interactions between variables collected.

\section{Definitions}

PPF recurrence was defined as the re-occurrence of ipsilateral pleural effusion after re-initiation of CAPD, which is diagnosed clinically with compatible pleural fluid biochemistry, CT peritoneogram or peritoneal fistulogram. Surgical complications were defined as complications requiring surgical intervention.

\section{Outcomes}

The primary outcome of interest is the recurrence of PPF. Secondary outcomes included surgical complications, need for surgical re-intervention and 30-day mortality.

\section{Ethics}

The study was conducted in accordance with the Declaration of Helsinki (as revised in 2013). The study is in progress of being reviewed by the Hospital Authority Clinical Research Ethics Review Board (KCC/KEC-20210069).

\section{Results}

From January 2009 to January 2019, there were 35 patients who underwent VATS management of CAPD associated PPF at the Department of Cardiothoracic Surgery, Queen Elizabeth Hospital, Hong Kong. The average age in this group was 60.8 years ( 44 to 82 years), there were 19 male patients $(54.3 \%)$. The average time from initiation 
Table 1 Illustration of patient parameters prior to and at the time of surgery

\begin{tabular}{lc}
\hline Parameter & Average value (\% or range) \\
\hline Age, years & $60.8[44-82]$ \\
Male & $19(54.3 \%)$ \\
Body weight, kg & $54.6[36-77]$ \\
Right side & $33(94.3 \%)$ \\
Average duration of CAPD prior to & $157.6[21-570]$ \\
pleural effusion, days & \\
Pre-existing diabetes mellitus & $10(28.6 \%)$ \\
Concurrent ACEl use & $3(8.57 \%)$ \\
\hline
\end{tabular}

CAPD, continuous ambulatory peritoneal dialysis; ACEI, angiotensin converting enzyme inhibitor.

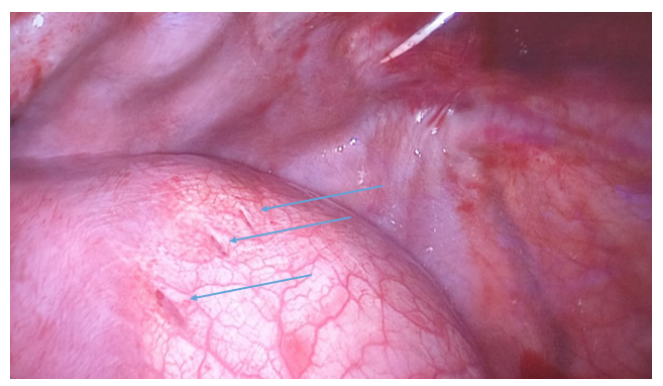

Figure 1 VATS view of small defects over the diaphragm (arrows). VATS, video-assisted thoracoscopic surgery.

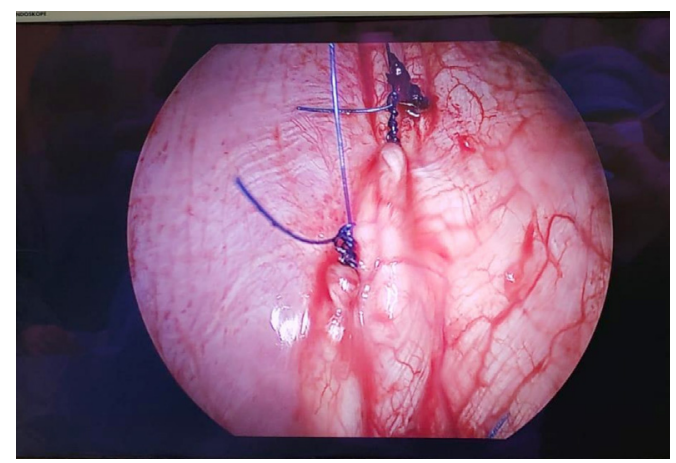

Figure 2 Closure of diaphragmatic defects using prolene sutures.

of CAPD to the first detection of pleural effusion was 157.6 days (21-570 days). Thirty-three patients (94.3\%) presented with right sided pleural effusion with the remainder presenting with left sided pleural effusions. The diagnosis of PPF was made in 14 patients $(40 \%)$ by peritoneal fistulogram, 11 patients $(31.4 \%)$ by corroborating clinical findings and in 10 patients (28.6\%) by CT peritoneogram (Table 1).

Surgical consultation was initiated upon detection of PPF and no other interventions were attempted prior to surgery in all patients. All patients received VATS pleurodesis with or without PPF repair. Mean operative time was 61 minutes (15-224 minutes). During thoracoscopic inspection of the diaphragm, 4 patients (11.4\%) had visually discernible diaphragmatic defects (Figure 1), 4 patients (11.4\%) had suspicious but non-confirmatory lesions over the diaphragm and 27 patients $(77.1 \%)$ had no PPF.

In respect to operative techniques employed, 15 patients $(42.9 \%)$ received prolene mesh mechanical pleurodesis alone, 12 patients $(34.3 \%)$ received concomitant mechanical pleurodesis and talc pleurodesis, 3 patients $(8.57 \%)$ received direct closure of their diaphragmatic defect (Figure 2) followed by mechanical and talc pleurodesis, 2 patients (5.71\%) received direct closure of their PPF followed by talc pleurodesis, 1 patient $(2.86 \%)$ received talc pleurodesis alone, 1 patient $(2.86 \%)$ received mechanical pleurodesis followed by tetracycline pleurodesis and 1 patient $(2.86 \%)$ required decortication due to dense adhesions surrounding the lung and pleural cavity. One patient developed haemothorax post-operatively requiring thoracotomy for haemostasis on post-operative day 1 . There were no 30 days mortalities.

Overall, the average time of suspension of CAPD was 176.2 days (35-589 days). Of the patients who were deemed suitable by nephrologists to re-initiate CAPD, the average time from operation to re-initiation of CAPD was 85.6 days (22-258 days). Of note, four patients were switched to long term HD without a trial of CAPD due to non-PPF related considerations after assessment by the nephrology team. Two of the excluded patients received mechanical pleurodesis alone while two of them received concomitant mechanical and talc pleurodesis.

In our series, the recurrence rate of pleural effusion in patients after re-initiation of CAPD was $25.8 \%$ (8 out of 31 patients). The average time to recurrence from re-initiation of CAPD was 32 days ( $0-181$ days) with 4 patients suffering immediate recurrence of pleural effusion. A variety of operative techniques were employed by the surgical team. Techniques included concomitant mechanical and talc pleurodesis, mechanical pleurodesis alone, talc pleurodesis alone, concomitant mechanical and tetracycline pleurodesis, decortication and direct closure with or without chemical pleurodesis. After reviewing the results, the authors decided to consolidate the operative techniques into concomitant 
Table 2 Comparison of surgical techniques in our patient group in terms of PPF recurrence against data taken from Chow et al. (1) for management of PPF with interruption of CAPD for 1-4 months followed by reinitiation

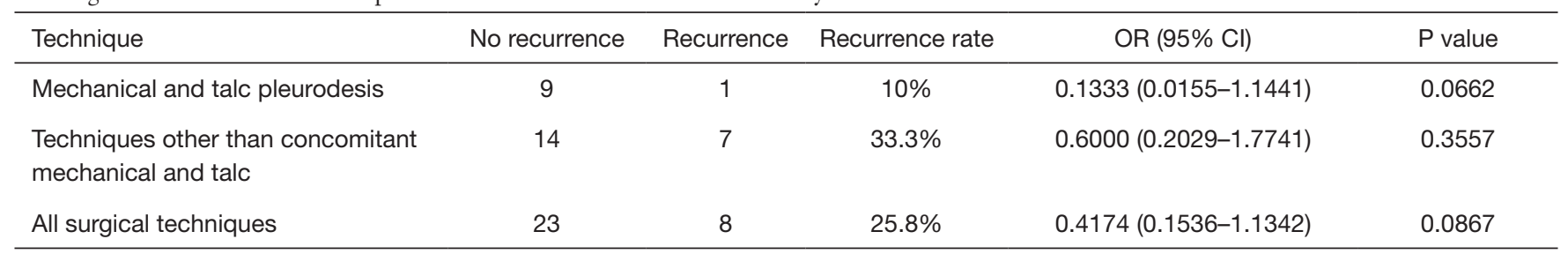

PPF, pleuro-peritoneal fistula; CAPD, continuous ambulatory peritoneal dialysis; OR, odds ratio; Cl, confidence interval.

Table 3 Comparison of surgical techniques in our patient group in terms of PPF recurrence against data taken from Chow et al. (1) for management of PPF with chemical pleurodesis via chest tube taken from Chow et al. (1)

\begin{tabular}{|c|c|c|c|c|c|}
\hline Technique & No recurrence & Recurrence & Recurrence rate & OR $(95 \% \mathrm{Cl})$ & $P$ value \\
\hline $\begin{array}{l}\text { Techniques other than concomitant } \\
\text { mechanical and talc and talc }\end{array}$ & 14 & 7 & $33.3 \%$ & $0.4706(0.1512-1.4648)$ & 0.1932 \\
\hline All surgical techniques & 23 & 8 & $25.8 \%$ & $0.4174(0.1536-1.1342)$ & 0.0867 \\
\hline
\end{tabular}

PPF, pleuro-peritoneal fistula; OR, odds ratio; $\mathrm{Cl}$, confidence interval.

Table 4 Comparison of surgical techniques in our patient group in terms of PPF recurrence against data taken from Chow et al. (1) for management of PPF with interruption of CAPD for 1-4 months followed by reinitiation and chemical pleurodesis via chest tube taken from Chow et al. (1)

\begin{tabular}{|c|c|c|c|c|c|}
\hline Technique & No recurrence & Recurrence & Recurrence rate & OR (95\% Cl) & $P$ value \\
\hline $\begin{array}{l}\text { Techniques other than concomitant } \\
\text { mechanical and talc and talc }\end{array}$ & 14 & 7 & $33.3 \%$ & $0.5405(0.1966-1.4863)$ & 0.2332 \\
\hline All surgical techniques & 23 & 8 & $25.8 \%$ & $0.3760(0.1498-0.9439)$ & 0.0373 \\
\hline
\end{tabular}

PPF, pleuro-peritoneal fistula; CAPD, continuous ambulatory peritoneal dialysis; OR, odds ratio; Cl, confidence interval.

mechanical and talc pleurodesis versus techniques other than concomitant mechanical and talc pleurodesis. This was due to the fact that concomitant mechanical and talc pleurodesis appeared to have the best result in terms of the lowest recurrence rate amongst surgical techniques at $10 \%$. On the other hand, consolidated results of techniques other than concomitant mechanical and talc pleurodesis yielded a recurrence rate of $33.3 \%$.

In an attempt to discern more insight from our data set, we have compared our operative techniques against historical data of treatment of CAPD associated PPF with non-surgical treatment taken from Chow et al. (1). Compared against historical data of the management of PPF by interruption of CAPD for 1-4 months followed by reinitiation, there was no statistically significant result obtained (Table 2). However when compared against historical data of chemical pleurodesis via chest tube (Table 3), results were significant for concomitant mechanical and talc pleurodesis with an odds ratio of 0.1046 (95\% CI, 0.0119-0.9213, $\mathrm{P}=0.0420)$. Furthermore compared to both methods of non-surgical treatments as detailed by Chow et al. (1), a statistically significant result was obtained when concomitant mechanical and talc pleurodesis was compared against non-surgical treatments with an odds ratio of 0.1201 (95\% CI, 0.0145-0.9946, P=0.0494). Furthermore, a statistically significant result was obtained when all surgical techniques were compared against non-surgical treatments with an odds ratio of 0.3760 (95\% CI, 0.1498-0.9439, $\mathrm{P}=0.0373$ ) (Table 4).

Analysis of age, gender, body weight at the of surgery, 
pre-existing DM, concurrent use of ACEI was not found to be statistically significant in regards to PPF recurrence by linear regression and Fisher's exact test. Pre-operative imaging for diagnosis of PPF was not statistically significant in predicting PPF recurrence. Presence of pre-operative diagnostic imaging did not constitute to a statistically significant increase in likelihood of being able to visualize the PPF intra-operatively.

There was no loss to follow up as all patients received CAPD or hemodialysis at Hospital Authorities, Hong Kong. Average duration of follow up was 1,594 days (111-4,096 days). All variables of concern were all able to be collected from the study subjects. 17 patients had died at the time of review, none of which had died as a direct result of surgical repair of their PPF. Of those who passed away, the average elapsed duration from operation to death was 1,119 days (111-2,221 days).

\section{Discussion}

CAPD is a form of renal replacement therapy performed by protocol set out by the renal team. CAPD is performed by the patient or the carer by instilling dialysate of various dextrose concentrations into the peritoneum through a dialysing catheter. The dialysate is allowed to dwell in the peritoneum for 4-6 hours, where the peritoneum acts as an interface for the exchange of fluid, electrolytes and toxins. After the prescribed time had elapsed, the fluid is then drained out from the catheter and a fresh bag of dialysate is infused. The summation is a constant presence of dialysate in the peritoneum which replicates the function of the kidneys (4).

CAPD is an efficacious and cost-effective mode of renal replacement therapy (5). As of 2013, it is estimated that there are 8510 patients requiring renal replacement therapy in Hong Kong, $76.2 \%$ of which are receiving peritoneal dialysis (5). As mentioned previously, the incidence of PPF is approximately $2 \%$ and its occurrence leads to the need to suspend CAPD with bridging haemodialysis until the definitive treatment of PPF (1).

The pathophysiology of PPF occurrence in the setting of CAPD is postulated to be due to a previously undiagnosed diaphragmatic defect, cyst formation or as a direct result of the pressure exert by the peritoneal dialysate on the diaphragm (3).

Non-operative means of pleurodesis has been documented, however its efficacy less well defined (6-8).

It appears that PPF recurrence is early with an average of 32 days after re-initiation of CAPD Notably 4 out of 8 patients suffered from pleural effusion immediately after re-initiation of CAPD. Close monitoring of each patient's response to CAPD in the early phase is necessary, as acute pleural effusion may not be well tolerated in this patient population.

Published case reports suggested that ACEI may induce pleural effusion (9). We postulate that ACEI usage may predispose patients to have recurrence of pleural effusion despite receiving VATS for CAPD associated PPF. However, ACEI use was not found to be statistically significant in predicting PPF recurrence in our patient series. Analysis of our data set against historical data taken from Chow et al. (1) showed that a dual technique of concomitant mechanical and talc pleurodesis is superior to chest tube pleurodesis in preventing PPF recurrence with an odds ratio of 0.1046. Furthermore, when compared to non-surgical management as a whole, all surgical methods, as well as concomitant mechanical and talc pleurodesis in particular was statistically significant in the prevention of PPF recurrence, with an odds ratio of 0.3760 and 0.1201 respectively. The authors postulate that the increased effectiveness of concomitant mechanical and talc pleurodesis in preventing PPF recurrence arises from the technical limitations in performing abrasive pleurodesis or pleurectomy to the diaphragmatic pleura, of which the culprit lesion is likely to be located. Consequently, talc slurry, owing to its fluid nature can be manipulated to coat the majority of the pleural cavity, including the diaphragmatic pleura. The authors believe that the effects of concomitant mechanical and talc pleurodesis creates stronger, more secure adhesions at critical areas than either technique can provide alone. We believe this explanation carries biological plausibility and thus generalizability as a surgical technique to be considered in the future. The above results are of particular note as there has yet been any surgical technique which had been described in literature which may reduce PPF recurrence that the authors are aware of.

The authors acknowledge that comparing our patients with a historical data set is a crude attempt in eluding more information from a relatively scarcely discussed topic in literature. The authors suggest that future effort should be aimed towards conducting a systematic review of current literature to consolidate the findings of the myriad of smaller scale studies available.

Some centres employ localization techniques such as injection of methylene blue into peritoneal dialysate or use of intra-operative pneumoperitoneum to assist the 
localization of a potential diaphragmatic defect during VATS $(10,11)$. However, this is not a routine practice at our centre. Further studies should be aimed towards addressing whether intra-operative confirmation of the site of leakage would decrease recurrence of PFF.

It is our routine practice to advise the nephrologists to withhold CAPD for at least 3 months after surgery to allow time for the fistula to heal. The rationale behind this suggestion is due to the mechanism of pleurodesis in inducing aseptic inflammation and thus approximation of the pleura through fibrosis, a process which requires time to mature. However, other series such as that published by Kumagai et al. and Shoji et al. suggest that CAPD can be restarted at an earlier date after surgery $(3,12)$. The exact safety period for CAPD suspension is yet to be determined and should be addressed by future studies.

One patient in our series suffered from haemothorax requiring thoracotomy for haemostasis. As uremic patients may suffer from platelet dysfunction, it should be made aware that proper correction of coagulopathy in uremic patients, such as use of DDAVP prior to surgery is important in reducing the risk of hemorrhagic complications from arising from the surgery (13).

This study is limited by its retrospective nature. The authors acknowledge that our sample size is still relatively small despite collecting a decade of data from a major thoracic surgery centre which serves multiple hospital clusters within Hong Kong. However, to the best of our knowledge, our series is already one of the largest documented in current literature Patients in this study were drawn from our surgical database. Undoubtedly selection bias is a key concern as there may have been patients with CAPD associated PPF who were not referred by nephrologists for cardiothoracic consultation. Furthermore, the portion of patient who were not offered surgery based on their underlying functional status or those who refused surgery were not accounted for. It is likely that the population deemed suitable to undergo surgery may be more physiologically fit, with fewer co-morbidities and better healing capabilities. The complication and recurrence rates derived from this study may be under-estimated.

\section{Conclusions}

Video assisted thoracoscopic surgery for treatment of CAPD related PPF is an effective and relatively safe procedure. Use of mechanical pleurodesis with concomitant talc pleurodesis appears to be more effective at decreasing
PPF recurrence.

\section{Acknowledgments}

Funding: This study was supported by the Department of Cardiothoracic Surgery, Queen Elizabeth Hospital.

\section{Footnote}

Reporting Checklist: The authors have completed the STROBE reporting checklist. Available at http://dx.doi. org/10.21037/jtd-20-3327

Data Sharing Statement: Available at http://dx.doi. org/10.21037/jtd-20-3327

Peer Review File: Available at http://dx.doi.org/10.21037/jtd20-3327

Conflicts of Interest: All authors have completed the ICMJE uniform disclosure form (available at http://dx.doi. org/10.21037/jtd-20-3327). The authors have no conflicts of interest to declare.

Ethical Statement: The authors are accountable for all aspects of the work in ensuring that questions related to the accuracy or integrity of any part of the work are appropriately investigated and resolved. The study was conducted in accordance with the Declaration of Helsinki (as revised in 2013). The study is in progress of being reviewed by the Hospital Authority Clinical Research Ethics Review Board (KCC/KEC-2021-0069).

Open Access Statement: This is an Open Access article distributed in accordance with the Creative Commons Attribution-NonCommercial-NoDerivs 4.0 International License (CC BY-NC-ND 4.0), which permits the noncommercial replication and distribution of the article with the strict proviso that no changes or edits are made and the original work is properly cited (including links to both the formal publication through the relevant DOI and the license). See: https://creativecommons.org/licenses/by-nc-nd/4.0/.

\section{References}

1. Chow KM, Szeto CC, Li PK. Management options for hydrothorax complicating peritoneal dialysis. Semin Dial 2003;16:389-94. 
2. Nakayama T, Hashimoto K, Kiriyama T, et al. Optimal imaging conditions for the diagnosis of pleuroperitoneal communication. BMJ Case Rep 2019;12:e228940.

3. Shoji F, Katsura M, Haratake N, et al. Surgical Repair of Pleuroperitoneal Communication with Continuous Ambulatory Peritoneal Dialysis. Thorac Cardiovasc Surg 2019;67:147-50.

4. Li PK, Chung KY, Chow KM. Continuous ambulatory peritoneal dialysis is better than automated peritoneal dialysis as first-line treatment in renal replacement therapy. Perit Dial Int 2007;27 Suppl 2:S153-7.

5. Leung CB, Cheung WL, Li PK. Renal registry in Hong Kong-the first 20 years. Kidney Int Suppl (2011) 2015;5:33-8.

6. Tatematsu S, Hosoya K, Ryuzaki M. Successful treatment of pleuroperitoneal communication with pleurodesis using autologous blood in a patient with severe heart failure undergoing peritoneal dialysis: a case report and brief literature review. Ren Replace Ther 2020. doi: 10.1186/ s41100-020-00273-0.

7. Benz RL, Schleifer CR. Hydrothorax in continuous ambulatory peritoneal dialysis: successful treatment with intrapleural tetracycline and a review of the literature. Am
J Kidney Dis 1985;5:136-40.

8. Chow KM, Szeto CC, Wong TY, et al. Hydrothorax complicating peritoneal dialysis: diagnostic value of glucose concentration in pleural fluid aspirate. Perit Dial Int 2002;22:525-8.

9. Kupeli E, Ulubay G, Ulasli SS, et al. Cilazapril-induced pleural effusion: A case report and review of the literature. Ann Thorac Med 2010;5:171-3.

10. Okubo T, Takahashi H, Kaneko Y, et al. Detection of the point of communication by pneumoperitoneum at the surgery for pleuroperitoneal communication. Kyobu Geka 2010;63:365-9.

11. Fang S, Wu Z, Wang Q, et al. Thoracoscopic surgery for management of pleuroperitoneal communication complicating continuous ambulatory peritoneal dialysis: A case report. Medicine (Baltimore) 2019;98:e15391.

12. Kumagai H, Watari M, Kuratsune M. Simple surgical treatment for pleuroperitoneal communication without interruption of continuous ambulatory peritoneal dialysis. Gen Thorac Cardiovasc Surg 2007;55:508-11.

13. Hedges SJ, Dehoney SB, Hooper JS, et al. Evidence-based treatment recommendations for uremic bleeding. Nat Clin Pract Nephrol 2007;3:138-53.
Cite this article as: Chen HYM, Chan HYH, Chan HMH, Cheung HL. Surgical management of pleuro-peritoneal fistula in chronic renal failure patient-safety and effectiveness. J Thorac Dis 2021;13(5):2979-2985. doi: 10.21037/jtd-20-3327 
Appendix 1 Data extracted with regards to PPF recurrence from Chow et al. (1) for management of PPF with interruption of CAPD for 1-4 months followed by reinitiation and chemical pleurodesis via chest tube

Interruption of CAPD for 1-4 months followed by reinitiation

- Recurrence: 20

- No recurrence: 24

- Recurrence rate $45.5 \%$

Chest tube pleurodesis

- Recurrence: 17

- No recurrence 16

Recurrence rate: $48.5 \%$ 